\title{
El perfil de la estructura narrativa de los audiovisuales animados chilenos 2000-2008
}

\section{The profile of the structural narrative in Chilean audiovisual animated films from 2000-2008}

\section{Felipe Silva Montellano}

Universidad Tecnológica Metropolitana

fsilvam@utem.cl

\begin{abstract}
Resumen El presente artículo expone los resultados centrales de la investigación FONDECYT de Iniciación, 11080150. Observamos los aspectos estructurales de la composición narrativa, mediante la lectura de los códigos formales dispuestos en la linea de tiempo, establecemos un primer estadio de observación de los mecanismos que articulan los códigos significantes del relato audiovisual, obteniendo un panorama general de los conocimientos compositivos que subyacen en los audiovisuales animados. Estas constantes y variables nos permite establecer relaciones de parentesco entre los perfiles formales de representación estructural. Concluimos que la comprensión de la naturaleza estructural, permite formular y sugerir un nuevo modo de enfrentar futuros proyectos de investigación transdisciplinarios.
\end{abstract}

Palabras clave Animación; estructura; composición; narrativa

Abstract This paper outlines the main results of the FONDECYT initial investigation 11080150. We observe structural and formal aspects of the narrative composition. By reading the formal codes in the timeline, we establish a first stage of observation and comparison of the mechanisms that articulate the significant codes in the audiovisual narrative, obtaining a general panorama of the compositional knowledge that underlies the films. These constants and variables allow us to establish relationships between the formal, structurally representative profiles. We conclude that comprehension of the structural and generic nature of the audiovisual animated films allows us to formulate and suggest a new way to tackle future interdisciplinary research projects related to audiovisual animated films.

Keywords Animation; structure; composition; narrative 


\section{Introducción}

Los estudios sobre los fenómenos de representación audiovisual que articula el mediocentrismo, generados desde el centralismo, como los de las realidades regionales y locales, tienen múltiples perspectivas, enfoques y objetivos de observación. Todos los trabajos referidos a los conocimientos sobre la utilización de símbolos en los relatos que modelan la imaginación colectiva, son potencialmente fecundos en relación al cine de imagen real.

Nuestra elección es centrarnos en un espectro cinematográfico de representación que expone universos de ficción, simbólicamente abstractos y cercanos al mundo de la fantasía de nuestra realidad nacional. Nos referimos al campo de los audiovisuales animados, universo del que aún hay mucho que explorar. En los estudios culturales contemporáneos relativos al universo de productos animados, no existen referentes sobre estudios formales y académicos que contemplen establecer una visión global sobre el proceso de vida completo, nos referimos a la gestión y preproducción, naturaleza física y objeto fílmico, como también la implementación y efectos en la sociedad. Según declara (Giroux, 2001), los estudios culturales suspenden y eluden el hacerse cargo de generar conocimiento sobre los productos de cine-animado generado desde el centralismo mediático, como de cualquier otra fuente local. Nuestra realidad nacional no es la excepción. Entendemos convencionalmente que los productos audiovisuales que pertenecen al mundo infantil, no tienen relevancia suficiente para levantar estudios revisionistas de los contenidos vertidos en ellos. Recordemos que existe una gran variedad de públicos objetivos identificados como receptores de productos audiovisuales animados, esto indica, con mayor razón, que debemos estudiar la audiovisualidad animada como un eje más de relaciones de discursivas, ya que este tipo de soporte también es portador de cargas significantes y contenidos valóricos.

Reconocemos los importantes aportes a los estudios comunicacionales previos a la presente investigación, los que, desde diferentes ángulos disciplinares buscan constatar el estado de la representación significante del panorama nacional, podemos decir que dichos trabajos desentrañan puntuales mecanismos que subyacen en los audiovisuales animados nacionales, (Cabello y Ortega, 2007), trabajo que relaciona filmes generados en realidades sociales originalmente distintas entre ellas y que pertenecen a una programación televisiva puntual, como también de los estudios que exploran los mecanismos semióticos entre productos audiovisuales que tienen razones objetivas $y$ naturalezas estructurales distintas (Del Villar, 2000-2008).

Sin duda alguna estas investigaciones son un importante aporte a la generación de conocimiento sobre el panorama audiovisual nacional. 


\section{La observación de las estructuras}

Nuestra propuesta es establecer, desde una perspectiva general, cuales son los conocimientos de los trabajos y proyectos generados desde la realidad chilena. Generamos una plataformas de análisis y de lectura, desarrollando una investigación, cuya primera consecuencia es establecer el estado de la situación del arte. De este modo los objetivos principales de la investigación se centran en comprender la naturaleza estructural y formal de los audiovisuales animados chilenos, para comenzar a erigir un sistema de observación de los productos audiovisuales animados que propicien una discusión crítica sobre este soporte comunicacional.

Inicialmente nuestro desafío fue trazar una estrategia de relación interdisciplinaria que explore, en este caso, conocimientos específicos que se desprenden de una lectura estructural formal, para establecer una visión panorámica del estado de las realizaciones creadas en los distintos ámbitos formales. Analizamos los proyectos profesionales generados mediante recursos establecidos por los organismos fuentes de financiamiento audiovisual. De igual forma abarcamos las iniciativas informales generadas por creadores independientes, los que están realizando de forma prolífica productos audiovisuales animados debido a la masificación de los medios tecnológicos de montaje digital. Debido a la naturaleza informal de estas últimas realizaciones, advertimos una especial dificultad a la hora de pesquisar y datar producciones de carácter independiente, ya que presentan particularmente una inaccesibilidad a las fuentes citadas, como también la ubicación críptica del estado de las realizaciones. Estos factores hicieron que parte de los documentos identificados aún no hayan sido recolectados y datado. Esperamos su incorporación a la base de datos que surge como consecuencia de esta investigación. Esta selección de audiovisuales animados y su consecuente lectura sistemática, no se ve afectada en los resultados por la falta de incorporación de aquellos proyectos no datados, como los que se generaron durante el proceso de la investigación, debido a que la muestra está sujeta al teorema central del límite por la gran cantidad de proyectos que conforman el cuerpo de documentos audiovisuales que estudiamos en la investigación recientemente desarrollada.

Para emprender el ejercicio de la lectura estructural nos basamos en el trabajo de Sless (2008), quien estudia la composición de los elementos visuales que configuran los textos de estudio escolares. En este estudio, afirma que la lectura de los componentes constitutivos de las piezas visuales, siguen un patrón gráfico definido y se puede comparar con la funcionalidad de la estructura de trasfondo de los textos. Esta composición genera un diagnóstico visual del recorrido sobre los contenidos cognitivos que el usuario ve u omite, asimilando 
los mecanismos de fijación de los conocimientos subyacentes en un determinado el relato. En nuestro caso hacemos un paralelo, correlativo al ejercicio planteado por Sless, aplicado a las estructuras mayormente complejas, como son los audiovisuales animados, además planteamos la hipótesis de que existe un patrón gráfico de la composición audiovisual que nos permite entender el comportamiento de los componentes estructurales. Entonces nos planteamos responder las siguientes preguntas sobre el tema de nuestra investigación ¿Existe una línea o perfil estructural de los audiovisuales animados?: Cuál es el perfil y patrón visual que arroja el análisis? ¿Cuáles son los conocimientos que subyacen en las líneas narrativas? ¿Cómo se comporta el flujo narrativo? ¿Son comparables, similares, entre ellos?

Entendemos que la multiplicidad de lecturas, sobre los diferentes códigos significantes de un producto audiovisual animado, es extremadamente abundante. Además, si consideramos las escalas de aplicación de dichas lecturas, las que pueden generar un universo cuantioso de asociaciones, que fácilmente se pueden encontrar en la esfera de la interpretación subjetiva, dificultando la individualización de elementos cualitativos y cuantitativos comunes entre los diferentes productos que conforman universo de muestras (Aumont, 1990). Por consiguiente, la elección de los componentes que configuran la forma fílmica de los audiovisuales estudiados, responde a la necesidad de eludir la contaminación de los datos obtenidos con las herramientas de observación. Esto quiere decir que mientras nos detengamos en los elementos y códigos significantes básicos, identificados desde la unidad fotograma, o la compleja relación de encuadres que podemos encontrar en una toma, y la suma de los códigos textuales, sonoros y visuales, quedamos imposibilitados de tener una visión global de todo el universo, como también lo declara (Metz, 19641972).

En la investigación realizada observamos las dimensiones de códigos estructurantes, los que son observables y cuantificables. En los códigos visibles cuantitativos observamos el uso de figuras retóricas dispuestas en la línea de tiempo, la distribución de los mitemas, el código pulsional y la frecuencia de división temporal. En los códigos visuales cualitativos apreciamos la representación icónica y la representación de género. En el presente artículo sólo nos centraremos en los resultado que derivan de la observación del comportamiento de los mitemas, en los cuales al analizar su disposición y orden, atribuimos un trazo lineal que usamos para comparar y correlacionar dichos componentes. Es un procedimiento de representación de hechos observables que usamos para responder las preguntas antes expuestas.

Elegimos la figura de los mitemas, ya que estos elementos, o unidades significantes, constituyen la estructura del relato y operan con claridad 
definiendo e identificando las etapas de desarrollo de una narración. Más aún, cuando hoy en día, el paradigma de la estructura narrativa básica, sigue siendo la visón aristotélica de construcción narrativa. Nos referimos a los distintos estados de evolución de los sujetos narrados en el relato: statu quo, desequilibrio, iniciación, búsqueda o viaje, clímax y el equilibrio o superación con retorno al statu quo equilibrio inicial o a un estado superior. Estos descriptores nos permiten tener una visión dinámica y certera de los elementos constituyentes del relato, como lo expondremos en adelante.

Formulamos un método de lectura de la dimensión espacio temporal. Este proceder está basado en los conocimientos propuestos por el diseñador Gui Bonsiepe, en lo que denomina retórica audiovisualística y visualización de conocimientos (Bonsiepe, 2005) Plantea un modelo deconstructivo, expone el cómo se posicionan los componentes significantes que constituyen la forma audiovisual, posibilitando su aplicación a múltiples objetivos de observación en investigación de análisis. Este procedimiento que probamos y reproducimos a nivel de laboratorio académico para ejercicios específicos de análisis de audiovisuales en la asignatura de Seminario de Expresión en la Carrera de Diseño de la Universidad Tecnológica Metropolitana, confirma que es un modelo idóneo para exponer diversos objetivos de lectura.

En la reciente investigación generamos un instrumento, el que denominamos Planilla de análisis, construido sobre la base de un gráfico cartesiano de doble entrada, con los ejes $X$ e $Y$. En el cual el eje $Y$ vertical, tiene todas las constantes que operan como objetivos identificables. En él disponemos todos los elementos constituyentes del relato descritos en los párrafos anteriores y mediante la lectura consistente de la observación reiterada y minuciosa, que se expresa en el eje de la línea de tiempo $X$ horizontal, posicionamos los elementos identificados. Usamos las medidas métricas de división del espacio, establecidas en las unidades mínimas que componen el relato audiovisual, desde las alturas, la unidad fotograma, escenas y secuencias del filme.

$\mathrm{Al}$ orden de los componentes elegidos, en este caso los mitemas, les atribuimos una forma lineal, un perfil o trazo que dibuja según las alturas en la dimensión $Y$ las etapas propias de la secuencia mitémica. Esta forma de representación de hechos observables, contienen las respuestas a las preguntas formuladas anteriormente sobre esta dimensión de la lectura.

Una vez leídas todas las muestras escogidas y trazados los perfiles que dibujan el flujo de cada elemento constituyente, nos dispusimos a observar los objetos audiovisuales en un mismo estadio de lectura, en un instrumento común para comparar y correlacionar dichos perfiles. Es así que alteramos 
intencionalmente el tamaño proporcional de la longitud cronométrica de todos los audiovisuales, para correlacionar y equipar las distintas propuestas leídas en los distintos niveles. Esta reducción del tiempo en una medida única, solo tiene la finalidad de comprender y apreciar las diferencias de los perfiles o trazos estructurales, como también conocer la correlación de la distribución espacial de los componentes de la narrativos. Esta alteración de las dimensiones no modifica la intención compositiva de cada muestra, como tampoco distorsiona las asociaciones textuales articuladas por los autores, en el interior de cada unidad significante de los relatos audiovisuales animados en cada filme.

Centrados únicamente en el universo disciplinar animado y, en específico, en aquellos audiovisuales animados lineales, como primera fase de la investigación realizada, entendemos que el cine, es en sí el gran nicho que alberga el lenguaje por el que se rigen todas las modalidades fílmicas de expresión, y asumimos el término audiovisual animado para abarcar, no sólo las realizaciones análogas que utilizan la creación de imágenes mediante la técnica cuadro a cuadro, sino más bien extendiendo dicha definición a aquellos proyectos que incluyen tecnologías digitales en los procesos intermedios. Incluimos en el descriptor a aquellas realizaciones que tienen distintos canales de difusión, como por ejemplo: el video digital, operas primas difundidas en festivales especializados y productos específicos televisivos como las seriales y los Clips Musicales. Es así que el marco referencial del universo de documentos que conforman el cuerpo de las muestras estudiadas, lo delimita el topos, audiovisuales chilenos y cronos, realizados entre los años 2000 y 2008, más la adjetivación de linealidad, que margina los productos audiovisuales interactivos para estudios posteriores.

Refiriéndonos al universo de las muestras, enunciamos que de un total de 102 filmes, 12 filmes sólo identificamos su existencia y no encontramos a los autores ni sus proyectos fílmicos, del universo inicial, 90 realizaciones fueron aportadas y cedidas directamente por sus autores. Del conjunto de muestras antes mencionadas, es decir, de las que registramos físicamente para observar, 70 audiovisuales conforman nuestras variables lógicas y, en definitiva, son los objetos analizados y leídos. Seis de las muestras son variables epistémicas con discriminabilidad, ya que muestran fertibilidad heurística, pues se abren a establecer nuevas relaciones dentro de las mismas variables, nos referimos a los seriales.

Encontramos un $12 \%$ del total de las muestras datadas, que son nuestras variables no relevantes confusionales, ya que se constituyen como cuerpo de nuevas investigaciones, estableciendo otro tipo de relaciones dentro de las mismas constantes y variables formuladas en el presente estudio, por ejemplo: 
los musicales, los documentales, los spots publicitarios y los experimentos académicos.

Observamos que el segmento de muestras estudiado, que particularmente denominamos lineal, enmarca múltiples géneros cinematográficos, es decir, señalamos y utilizamos como descriptores de conjuntos individualizados: lo narrativo, lo artístico expresivo, los proyectos seriados y los productos musicales. Entre los cuales identificamos: drama, comedia, ficción. Como también con sus correspondientes adjetivaciones: drama ficcional, drama histórico, ficción étnica, comedia ficción, ficción artística, ficción erótica artística, revisionismo etnográfico, etc. El género documental no está presente en este universo datado de audiovisuales animados.

Nuestro instrumento de análisis confirma que los audiovisuales animados pueden ser observados y sometidos a un estadio de lectura.

La lectura de las muestras indicaron que existe un línea constante y visible presente en todas las propuestas audiovisuales, y de comienzo a fin presentan un carácter oblicuo ascendente, esto muestra una intención evolutiva de un estado $A$, comienzo a otro B, término. Este carácter progresivo está presente, como un trasfondo de todas las variantes, en la disposición de los puntos de inflexión del relato de la línea que conforma el desarrollo entre ambos estados. Nos referimos a que esta evolución es un trayecto que aumenta las intención cualitativa de los componentes formales del filme y no necesariamente significa una evolución positiva en el desarrollo textual.

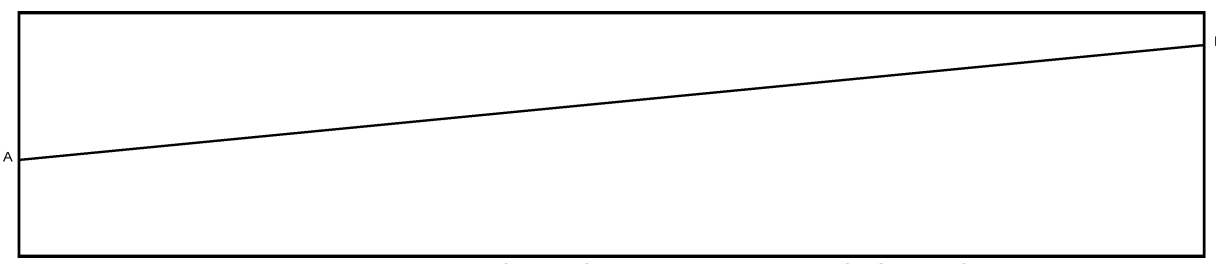

Esquema 1. Línea constante de evolución narrativa en la línea de tiempo.

Nuestras lecturas indican que el $33 \%$ con 23 realizaciones del total de las muestras, disponen todos los mitemas, 18 de ellos en el mismo orden secuencial mencionado con anterioridad y cinco muestras alteran y repiten algunos elementos, por ejemplo, incluyen dos desequilibrios o clímax. Así mismo encontramos un $36 \%$ con 25 muestras, que exponen cinco mitemas. Observamos que en este conjunto hay omisión de un elemento constituyente, que se alterna entre la iniciación y el equilibrio final. Esquema 3 y Gráfico 1. 
Ambas visiones, que suman un total de $69 \%$, indican una fuerte y marcada tendencia de configuración argumental correlativo al paradigma aristotélico. Esto nos lleva a inferir que existe una inclinación natural e inconsciente de representación discursiva de carácter conservador desde el punto de vista occidental. Identificamos en este universo de muestras una transversalidad de aplicaciones en el plano genérico, ya que, por ejemplo, tenemos filmes de la categoría expresiva-artística que tienen esta construcción paradigmática.

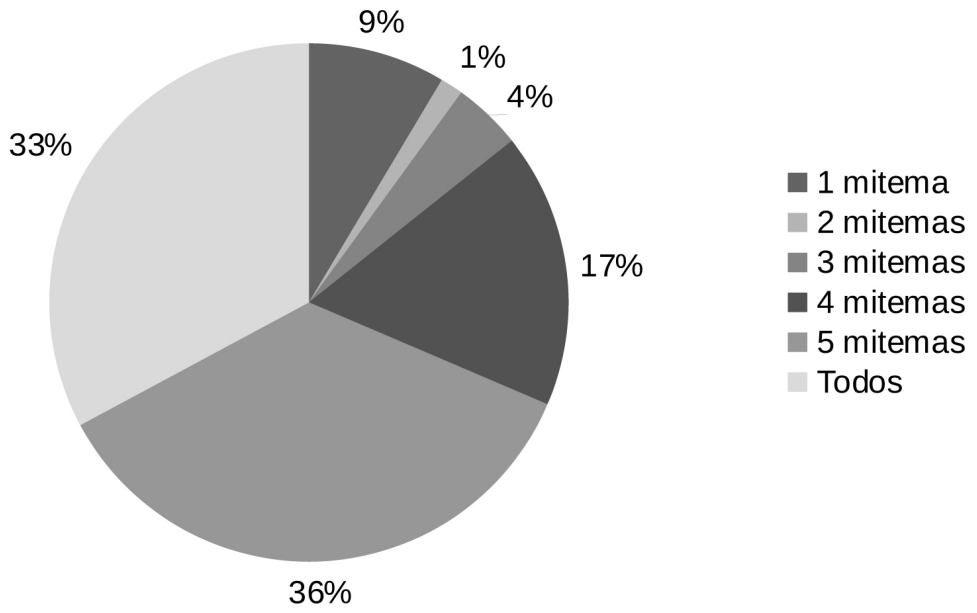

Gráfico 1. Distribución porcentual del uso de mitemas en la línea de tiempo.

En la observación de todas las líneas de lectura, encontramos que dos áreas del segmento horizontal marcan con claridad los puntos de inflexión del relato. Estos recursos mitémicos corresponden al desequilibrio y clímax y tienen un carácter flexible, se expanden y contraen variando el trazo del perfil en la estructura audiovisual. Por consiguiente, todos los desequilibrios coinciden en una zona común cercana al inicio de los audiovisuales, la que llamamos la zona de desequilibrio. Así mismo, todos los clímax coinciden en una zona cercana al término de los audiovisuales, la que llamamos la zona de clímax. En resumen, hay un claro y marcado perfil o patrón repetitivo que varía en las zonas de inflexión, desequilibrio y clímax. Esquema 2.

A diferencia del enunciado anterior, apreciamos que el recurso viaje es una constante en el universo total de los audiovisuales estudiados, siempre está presente. También advertimos que la sección correspondiente al viaje posee diversas inflexiones menores que representan las etapas de superación previas al clímax. Esquema 3. 
Observamos que cinco realizaciones argumentales proponen nuevos paradigmas en el orden de la estructuras, principalmente en la repetición de componente: dos desequilibrios, dos iniciaciones, dos clímax, o bien terminar después de un clímax con un desequilibrio.

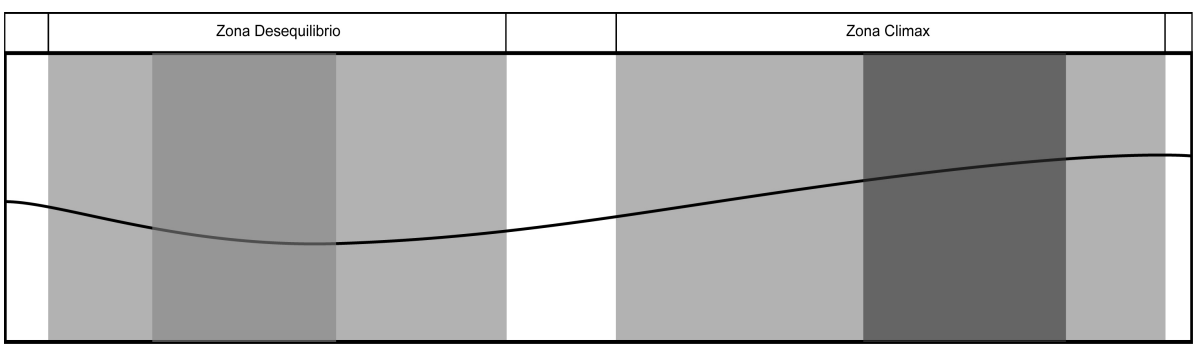

Esquema 2. Distribución de zonas comunes de los puntos de inflexión del relato en la línea de tiempo.

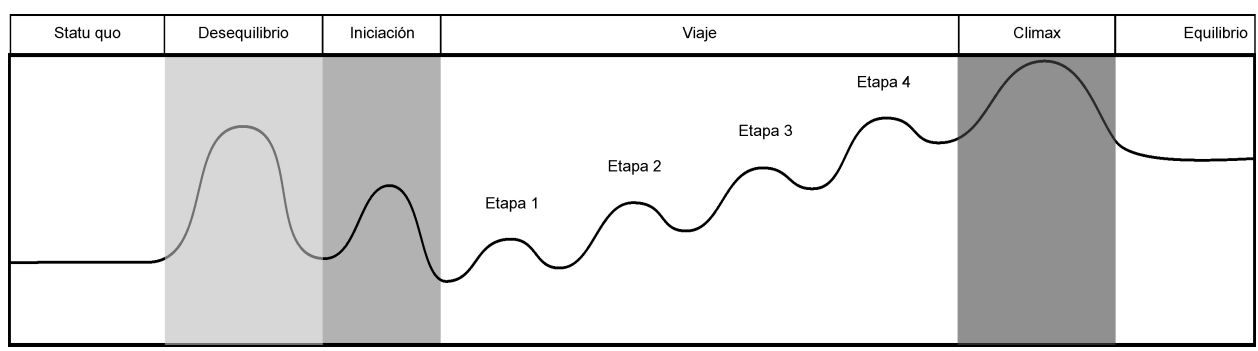

Esquema 3. Distribución secuencial del uso de mitemas en la línea de tiempo.

Las muestras indican propuestas narrativas provenientes de las artes visuales, y que en esta investigación, denominamos artístico experimental. Muestran una alteración del orden y disposición de los elementos constitutivos de la narración, como también la omisión de componentes. Tenemos el caso extremo en que seis audiovisuales utilizan el viaje como la única estructura que sustenta la propuesta audiovisual, y se configura como una sucesión continua de acontecimientos narrados que carecen de una razón, es sólo una progresión de hechos ascendente. Esquema 4. Siguiendo el mismo paradigma, un sólo audiovisual incluye únicamente el clímax como conclusión final. Esquema 5.

Encontramos dos propuestas provenientes del mundo de la expresión artística, que conforman parte de las variables relevantes y que también poseen estructuras completa en la cantidad y disposición del paradigma aristotélico.

Estas muestras indica que este universo audiovisual, no posee una planificación consciente de las nuevas visiones estructurales y narrativas. 
Mediante una entrevista directa con los autores, detectamos que la realización tiene un carácter de desarrollo fortuito y son resultado de la experiencia expresiva, en la cual la planificación básica, mediante el uso de story-board, puede o no estar presente, actuando únicamente como una referencia a la propuesta visual. Es así que el desarrollo surge de manera espontánea junto con el avance técnico, propio de la creación. Observamos que esta naturaleza instintiva, espontánea y a veces fortuita, trasciende los universos de muestras. Sólo un autor declara que sí piensa con detención la estructura, a diferencia de los demás, quienes dan por natural y obvio que el flujo estructural debe comportarse de esa manera. No hay una conciencia reflexiva del desarrollo de la composición narrativa, más bien es una condición tácita.

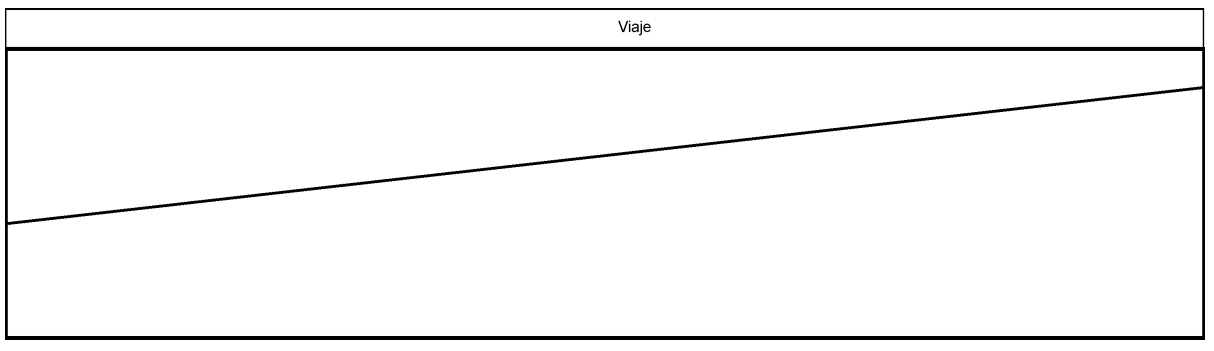

Esquema 4. Distribución secuencial donde predomina el viaje como única estructura central.

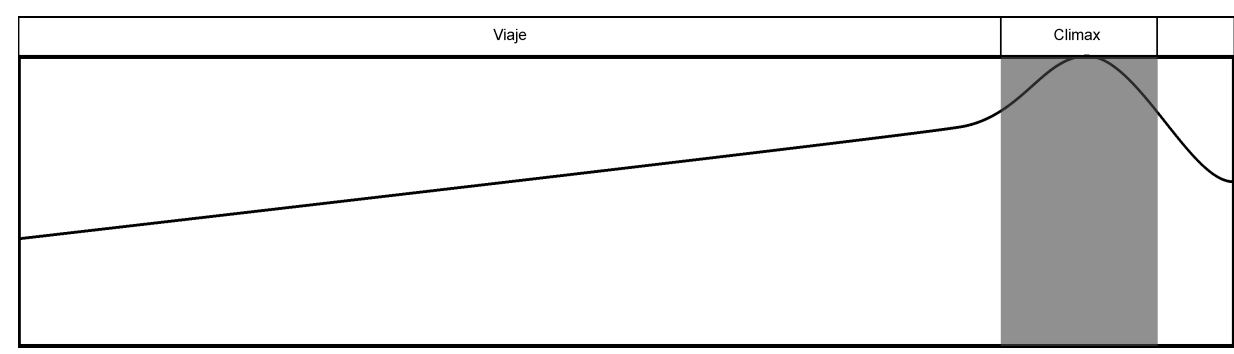

Esquema 5. Distribución secuencial donde predomina el viaje como estructura central incorporando sólo el climax como punto de quiebre.

Debemos destacar que dos de las dimensiones de lectura, el código pulsional y la frecuencia escénica, cuyos sus resultados no exponemos en este artículo, tienen estrecha relación con la dimensión de lectura de los mitemas. Enunciamos que: el código pulsional representado mediante una escala simple de actividad visual, coincide con los puntos de inflexión del relato, ya que concentran mayor actividad con las zonas de inflexión de la narración 
desequilibrio y clímax. De la misma forma, el ritmo escénico, es decir la frecuencia de segmentos en la división y fragmentación del espacio tiempo, en este caso las escenas. También coinciden en señalar mayor concentración y frecuencia en los principales puntos de inflexión de la actividad mitémica en las zonas, desequilibrio y clímax.

Este hecho indica que la reproducción de la lectura, mediante diferentes indicadores dentro de cada filme, presentan rasgos sincrónicos y coincidentes. En estos casos los puntos de tensión argumental, nos corrobora que la aplicación de la lectura está bien ejercida sobre la dimensión mitémicas.

En otra dimensión de lectura, la disposición de las figuras retóricas no tiene correlación ni coincidencia con las tres dimensiones antes señaladas, infiriendo que la composición estructural es independiente de la forma significante de representar. Las figuras retóricas utilizadas por los diferentes autores no son predeterminates a la aplicación de los mitemas encontrados, por ejemplo, para representar un desequilibrio, los autores pueden utilizar una metáfora o un flash-back.

Debemos mencionar que las variables no relevantes confusionales de esta investigación, que también consideramos audiovisuales animados narrativos lineales, poseen una estructura que las excluye de la presente lectura analítica, nos referiremos primero al género musical. Observamos que los Clips musicales son narraciones que están supeditadas al texto de la canción, la representación fílmica es textual al código sonoro, y poseen una puesta en escena cuya estructura recurre a la reiteración de secuencias modulares, en este caso los estribillos. Estos generan una constante repetición de escenas y secuencias, modificando la linealidad del relato. Enunciamos que cada estructura estribillo en sí, es un componente que devela o adelanta el carácter del clímax final, incluso comportándose como pequeños clímaxintermedios. Esta particularidad del género, se convierten en un motivo consistente para realizar una nueva exploración puntual y particular, que considera los elementos constructivos del género como una determinante en la naturaleza y condición estructural de este universo. Esquema 6.

De la misma forma podemos levantar otro análisis preliminar con el universo de los spots publicitarios, los que también son audiovisuales animados narrativos lineales, articulando una estructura dinámica centrada en la persuasión, donde el producto de consumo es la razón del clímax final. La narrativa se constituye de un desarrollo simple comenzando en un quiebre del statu-quo mediante un desequilibrio que justifica el uso del producto con un viaje breve, generalmente asociado a la figura del gag, que junto al clímax configuran el pack final, figura en la que se exhibe físicamente el producto o servicio 
ofrecido, presentando además, en algunos casos, un estado de equilibrio posterior al pack. Al igual que el universo anterior este conjunto califica para ser analizado dentro de su contexto. Esquema 7.

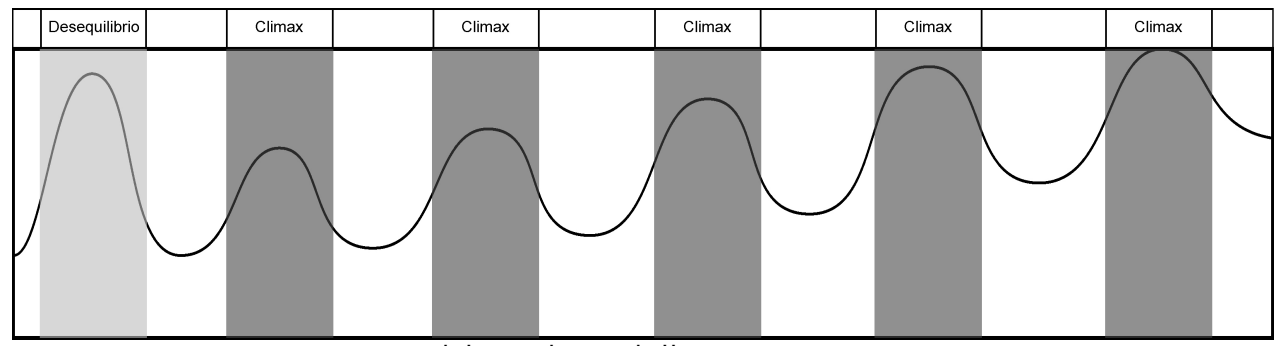

Esquema 6. Resumen del uso de estribillos como componentes reiterativos.

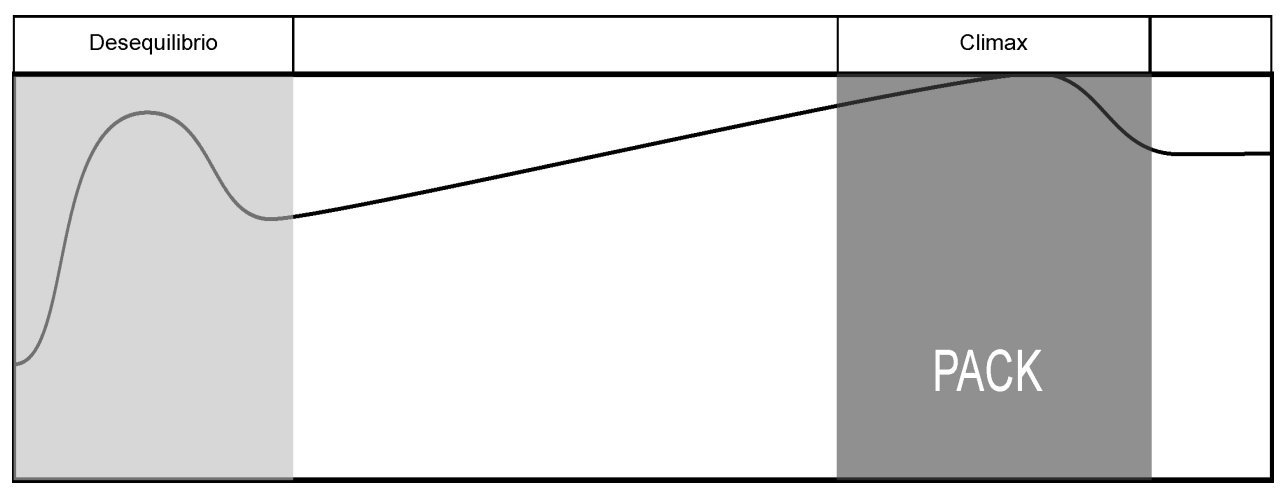

Esquema 7. Resumen del carácter dinámico del Spot publicitario.

$\mathrm{Al}$ igual como nos referimos en los párrafos anteriores, creemos que el universo de los audiovisuales seriado merecen ser estudiados en su conjunto, la razón de la estructura serial que en sí se rige bajo el perfil expuesto en el esquema 1. implican una serie de mecanismos definidos por la Biblia propia de cada proyecto, que predetermina el actuar compositivo de recursos significantes. Creemos que este universo debe ser estudiado comprendiendo la totalidad de los capítulos de la serie e individualizando cada proyecto como un subuniverso de relaciones establecidas. Debemos destacar que en el caso de los proyectos seriados de Chile, existe un tratamiento temático común. Del universo observado, el $100 \%$ de los audiovisuales animado seriales, a nivel de representación formal, abordan el tratamiento de los personajes en un mismo rango etáreo, con personalidades heterogéneas y complementarias, que se 
desenvuelven en un entorno común y que colectivamente resuelven en el viaje un problema desencadenado por un desequilibrio inicial.

\section{Conclusiones}

Este estudio genera un avance, en lo que respecta la ejecución y puesta en práctica de la lectura estructural de los audiovisuales animados. Creemos que el instrumento planilla de análisis es una herramienta dinámica en su aplicación, independiente de las realidades, escalas niveles de observación $y$, particularmente, para exponer análisis aún más precisos y específicos si reducimos el foco de observación y el universo de muestras.

Nuestras observaciones indican que una gran mayoría de audiovisuales animados se estructuran sobre la base de una dependencia del paradigma centralista, en contraposición al surgimiento de nuevos lenguajes y conocimientos de realidades locales.

Estos resultados indican que, tanto las variables relevantes, como las no relevantes que conforman el cuerpo de muestras del presente análisis, poseen un perfil basal común, que nos hace inferir que existe una relación de parentesco estructural, este es un importante hallazgo que nos lleva a proponer un primer esbozo de clasificación, y sin tener la intención de forzar una investigación en estudios culturales utilizando los paradigmas de las ciencias naturales, queremos aproximarnos a proponer una clasificación taxonómica que describa y unifique el universo total de la realidad del soporte animado.

Concluimos que existen diferencias sustanciales y manifiestas en la articulación de los componentes de las realizaciones animadas, que determinan e identifican un objeto audiovisual animado con el correspondiente público objetivo. La forma estructural es el reflejo de las características funcionales del objeto estudiado, ya que la articulación de elementos constituyentes, devela una correlación con el formato físico y la estrategia de distribución de las realizaciones animadas nacionales.

Sugerimos que al formular nuevos proyectos de análisis, se deben considerar las diferencias estructurales de formato entre productos audiovisuales animados, pues para profundizar en el estudio de los mecanismos significantes, es necesario reflexionar y reconocer de manera consciente las singularidades estructurales de este universo audiovisual.

Los resultados nos dan directrices que permitirán diseñar nuevas estrategias en el campo de la investigación básica, como por ejemplo: la toma de conciencia de los organismos encargados del fomento audiovisual de productos animados, en torno al manejo y control ético de las influencias predominantes en la creación y construcción de idearios e identidades. 
En el campo de la investigación aplicada, concluimos que nuestros resultados ofrecen a los autores la visión de adquirir conciencia autocrítica y reflexiva en la generación de propuestas audiovisuales, pensadas desde la estructura, fundamentalmente si nos referimos a la búsqueda de nuevos lenguajes expresivos que alternen visiones en los paradigmas discursivos actuales de representación audiovisual.

\section{Referencias bibliográficas}

Altman, R. (2000). Los géneros cinematográficos. ( $3^{\circ} \mathrm{ed}$.). Barcelona: Paidós.

Aprile, O. (2008). La Publicidad audiovisual. (1 ํed.). Argentina: La Crujía.

Aumont, J. y Marie M. (1990). Análisis del filme. (2ed.). Barcelona: Paidós.

Barker, C. (2003). Televisión, globalización e identidades culturales. ( $3^{\circ}$ ed.). Barcelona: Paidós.

Bonsiepe, G. (2005). "Retórica audiovisualística y visualización de conocimientos". Santiago: Ciclo de conferencias Universidad Tecnológica Metropolitana, Junio.

Bordwell, D. (1995). El Significado del filme, Inferencia y retórica de la interpretación cinematográfica. $\left(2^{\circ}\right.$ ed.). Barcelona: Paidós.

Buxó, M. y de Miguel, J. (1999). De la investigación audiovisual. (1 ed.). España: Proyecto a.

Cabello, P.y Ortega, C. (2007). "Las relaciones de género en los dibujos animados de la TV chilena”. Cuadernos de Información. № 21. Obtenido en octubre del 2010 en http://redalyc.uaemex.mx/src/inicio/ArtPdfRed.jsp?iCve=97117398005.

Casetti, F. y Di Chio, F. (1998). Análisis del film. (3 ed.). España: Paidós

Del Villar, R. (1997). Trayectos en semiótica filmico televisiva. ( $1^{\circ}$ ed.). Santiago: Dolmen.

Gaudreault, A. y Jost, F. (1995). El relato cinematográfico, ciencia y narratología. (2ed.). España: Paidós.

Giroux, H.A. (2001). El Ratoncito Feroz, Disney o el fin de la Inocencia. ( $2^{\circ}$ ed.). España: Fundación Germán Sánchez Ruiperez.

Margolin, V. (2005). Las Rutas del Diseño: estudios sobre teoría y práctica. ( $\left.1^{\circ} \mathrm{ed}.\right)$. Buenos Aires: Nobuko.

Metz, C. (2002). Ensayos sobre la significación en el cine. Vol I y II 1964-1972. (4ed.). Barcelona: Paidós.

Sless, D. (2005). "Information Design: a functional analysis of three chilean textbooks". Santiago: Seminario de Comunicación Visual para Libros de Texto e Hipertexto. Unidad de Currículum y Evaluación del Ministerio de Educación. 5 y 6 de mayo.

Silverstone, R. (2010). La moral de los medios de comunicación. ( $1^{\circ}$ ed.). Argentina: Amorrortu Editores. 
Shohat, E. y Stam, R. (2002). Multiculturalismo, cine y medios de comunicación. ( $\left.3^{\circ} \mathrm{ed}.\right)$. España: Paidós .

Van Dijk, T. (2008). El discurso como estructura y proceso. ( $\left.3^{\circ} \mathrm{ed}.\right)$. España: Gedisa 